\title{
Further Evidence for a Dissociation Between Different Forms of Mnemonic Expressions in a Mouse Model of Age-related Cognitive Decline: Effects of Tacrine and S 17092, a Novel Prolyl Endopeptidase Inhibitor
}

\author{
Aline Marighetto, ${ }^{2}$ Khalid Touzani, Nicole Etchamendy, Cedric Cortes \\ Torrea, Guillaume De Nanteuil, ${ }^{1}$ David Guez, ${ }^{1}$ Robert Jaffard, and Philippe Morain ${ }^{1}$ \\ CNRS - UMR-5106, Laboratoire Neurosciences Comportementales and Cognitives, 33405 Talence Cedex, France; and ${ }^{1}$ Institut de Recherches \\ Internationales Servier, 92415 Courbevoie Cedex, France
}

\begin{abstract}
It has been demonstrated previously on the radial maze that the emergence of an age-related mnemonic impairment is critically dependent on the form which the discrimination problems took. Hence, when the arms were presented one by one (i.e., successive go-no-go discrimination), both adult and aged mice learned to distinguish between positive (baited) and negative (unbaited) arms readily, as evidenced by their increased readiness to enter positive relative to negative arms (i.e., by a differential in arm-entry latencies). A selective impairment in the aged mice was seen when these arms were presented subsequently as pairs, such that the mice were confronted with an explicit choice (i.e., simultaneous 2-choice discrimination). When discriminative performance was measured by the differential run speed between positive and negative arms, aged mice were also impaired. This was particularly pronounced in the 2-choice discrimination condition. We examined the effects of tacrine $(3 \mathrm{mg} / \mathrm{kg}$, subcutaneously) or S $17092(10 \mathrm{mg} / \mathrm{kg}$, orally) in aged mice on the three behavioral indices of this 2-stage spatial discrimination paradigm. The results indicated that: (1) Tacrine, but not S 17092, enhanced the acquisition of go-no-go discrimination as reflected in arm-entry latencies; (2) both drugs improved choice accuracy in simultaneous discrimination, although the effect of tacrine was less striking and, in particular, far from statistical significance in the very first 2-choice responses; and (3) neither drugs significantly affected run-speed performance. We conclude further that the specific patterns of drug effects on the three indices of discriminative performance might suggest that each index is associated with a distinct form of mnemonic expression relying on separate neural systems.
\end{abstract}

In humans, declarative/explicit memory appears to be more vulnerable to deterioration in senescence than procedural/ implicit memory (Poon 1985; Gabrieli 1996; Schugens et al. 1997). One cardinal characteristic of declarative memory is its flexibility as exemplified by its capacity to support inferential use of memories in novel situations (Cohen 1984). Using a two-stage paradigm of discrimination learning, Marighetto et al. (1999) have previously demonstrated that aged mice displayed impaired inferential abilities when they were required to make an explicit choice between two eventualities that were only encountered before separately, but never conjointly. In Stage 1, the mice learned to discriminate between the valence of three baited (positive) and three unbaited (negative) arms in a radial maze with each of the arms presented one at a time, i.e., successive

${ }^{2}$ Corresponding author.

E-MAIL a.marighetto@neurocog.u-bordeaux.fr; FAX 33-55684-8743. go-no-go discrimination. Successful discrimination was indicated by the animals' increased readiness to enter positive arms relative to negative ones. In Stage 2, the animals were confronted with an explicit choice between one positive arm and one negative arm they had learned to discriminate previously. Aged mice, but not young ones, were unable to translate their preference for the positive arm shown in Stage 1 into a correct choice in Stage 2.

In this paradigm, the presence or absence of a mnemonic impairment in the aged mice is critically dependent on the different forms that a discrimination problem can take. One interpretation is that these corresponded to two forms of memory expression for the same piece of previously acquired information, and that only one is impaired in the aged mice. Such inflexibility of mnemonic expression has been proposed as a mouse model of age-related declarative memory decline in humans. Indeed, it represents a specific alteration in the ability to compare and contrast information originating from separate sources and this ability has

LEARNING \& MEMORY 7:159-169 @ 2000 by Cold Spring Harbor Laboratory Press ISSN1072-0502/00 \$5.00

$$
\begin{array}{lllllllllllllll} 
& E & A & R & N & I & N & G & \mathbf{Z} & M & E & M & O & R & Y \\
\text { www.learnmem.org } & & & &
\end{array}
$$


been considered as a cardinal characteristic of human declarative memory (Cohen 1984). Furthermore, the specificity of this deficit is consistent with the relational theory of hippocampal function (Eichenbaum 1992; Eichenbaum et al. 1994). According to this theory, the neural substrate underlying memory for separate pieces of information (as in successive go-no-go discrimination) is distinct from the hippocampal-dependent one that sustains the construction and storage of relational representations of past experiences. Flexible deployment of memories (as exemplified in the explicit 2-choice discrimination) is critically dependent on the integrity of the latter system centred on the hippocampal formation.

We believe that this mouse model of the preferential loss of declarative memory seen in human senescence might be of particular relevance to preclinical evaluation of potential pharmacological interventions against age-related cognitive disorders. Because the basic components involved in the two stages are essentially identical (in terms of the stimuli presented, the responses required, motivation, and reward magnitude), this design should enable one to evaluate "direct" effects of drugs on cognition without confounding from nonspecific (motivation, affect) factors that could "indirectly" affect cognitive performance. This design should also enable one to discriminate among procognitive drugs, those that specifically affect a form of memory that is altered in our aged subjects from those that possess a more general facilitatory effect on memory. Therefore this model might be useful in selecting from among alternative development candidates. It could also be helpful in evaluating potential mechanisms of cognitive impairment with consequent implications for directing future therapeutical approaches.

The primary objective of our study was to assess the potential value of this novel paradigm in pharmacological research. To this end, we examined the effects of two potential pro-cognitive agents, each targeted at a separate neurobiological process, on this mouse model of age-related cognitive decline.

The first drug, tacrine, was selected here as it represents one of the most popular agents designed to augment cholinergic transmission, a therapeutic route motivated by the classical cholinergic hypothesis of cognitive decline in aging. Supports for this hypothesis have derived from evidence of a cholinergic deficiency in aged animals and humans, as well as in age-related pathological conditions such as Alzheimer's disease (for a review, see Bartus et al. 1985; Gallagher and Colombo 1995). This has led to the development of cholinomimetics as potential therapeutic agents, designed to compensate for the atrophy or loss of basal forebrain cholinergic neurones seen in senescence (Bartus et al. 1985) and related pathologies (Davies and Maloney 1976; Coyle et al. 1983). Among such cholinergic agents, the cholinesterase inhibitor, tacrine, has generated consid- erable preclinical (for review, see Mohammed 1993) and clinical interests (Qizilbash et al. 1998). Here, we adopted a chronic treatment regime of this drug that has been shown previously to be effective in ameliorating memory performance in rodents (Flood and Cherkin 1988; Itoh et al. 1990; Riekkinen et al. 1991; Kirkby et al. 1996; Pavone et al. 1998).

However, clinical trials have shown that the efficacy of cholinomimetics is often limited and their therapeutic window is relatively narrow. These limitations highlighted the need for the development of alternative pharmacological approaches. Potential targets for novel therapeutic intervention include factors that might influence the activity and/or survival of the cholinergic system as well as other neurotransmitter systems known to undergo alteration during aging. S 17092, the second drug selected in our study, represented such a drug in this category.

S 17092 is a novel inhibitor of prolyl endopeptidase (PEP, EC3.4.21.26). PEP is a member of the serine-protease family that hydrolyzes peptide bonds at the L-proline carboxy terminal. PEP plays an important role in the catabolism of proline-containing peptides such as substance $\mathrm{P}$, arginin-vasopressin, thyrotropin-releasing hormone (TRH), bradykinin, angiotensin, neurotensin, and oxytocin (Wilk 1983). Hence, the action of $S 17092$ is expected to retard the degradation of a wide range of neuroactive peptides. There are a number of hypotheses suggesting that pharmacological interventions targeted at neuropeptides might be effective treatment for age-related decline.

Firstly, the neuropeptide TRH (among others) has been shown to promote cholinergic function and the release of acetylcholine (Giovannini et al. 1991; Bennett et al. 1997). Secondly, it has been demonstrated that several neuropeptides can promote processes related to functional recovery following CNS damage (Dekker et al. 1987; Pitsikas et al. 1991; Mattioli et al. 1992; Lestage et al. 1998a) and display neurotrophic actions in vitro (Iwasaki et al. 1989; Whitty et al. 1993). Thirdly, a number of neuropeptides are also found to be reduced in aged animals and humans (Buck et al. 1981; Dupont et al. 1981; Wang et al. 1981). For example, deficiencies in substance $P$ and vasopressin have been reported in postmortem studies of cerebral tissue derived from patients with neurodegenerative diseases (Ferrier et al. 1983; Rossor et al. 1986; Beal and Mazurek 1987; Husain and Nemeroff 1990). Hence, peptidergic alterations might play a significant role in age-related cognitive decline.

This suggestion is further supported by evidence for a beneficial effect on mnemonic performance following substance $P$ treatment in aged rats (Hasenöhrl et al. 1990). A similar facilitation has also been seen in patients with Alzheimer's disease following TRH treatment (Mellow et al. 1989,1993; Molchan et al. 1992) and in volunteer subjects treated with scopolamine (Molchan et al. 1990,1992). However, the effectiveness of systemic neuropeptidergic treat-

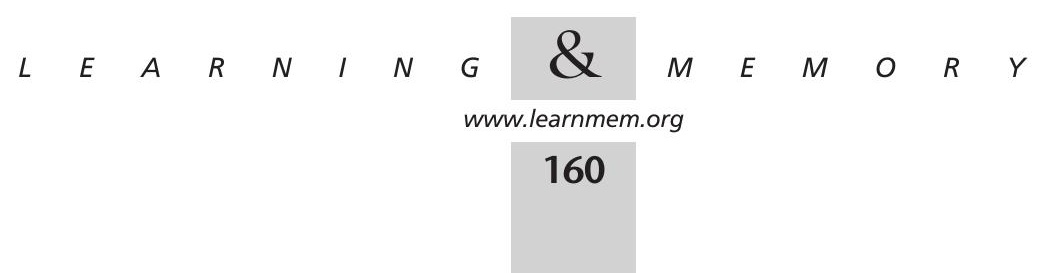


ment is generally viewed with scepticism because of the peptides' relative impermeability through the bloodbrain barrier. This is further complicated by the possibility that not one but several neuropeptides might be involved in mnemonic decline associated with aging. Therefore, an agent such as S 17092 that can increase the brain levels of several neuropeptides concomitantly might provide a promising approach. Chronic administration of $\mathrm{S}$ 17092 in rodents has been shown to possess beneficial effects on different forms of memory performance (Lépagnol et al. 1996; Lestage et al. 1998b). We further investigated the procognitive effects of this compound at a PEP inhibitory dose ( $50 \%$ inhibition of cortical PEP activity; Portevin et al. 1996) that has been reported to be behaviorally effective in aged mice (Lépagnol et al. 1996; Lestage et al. 1998b).

The results described here have been previously presented in an abstract form.

\section{RESULTS}

\section{Stage 1}

\section{Response Latency}

All mice, except two from the vehicle group (which were rejected from further testing and excluded from any analysis) reached the criterion performance required for transition from Stage 1 to Stage 2. As shown in Figure 2 , tacrine-treated mice appeared to require less training to attain criterion performance compared to the other two groups. A randomized block oneway ANOVA on the number of sessions to criterion yielded a significant Group effect $\quad[\mathrm{F}(2,34)=3.649 ; \quad P=0.04]$. However, post-hoc pair-wise comparisons revealed that the difference between the tacrine group and each of the two others failed to reach statistical significance [tacrine vs. vehicle: $P=0.068$; tacrine vs. S 17092 : $P=0.116$; vehicle vs. $\mathrm{S} 17092: P=0.82]$.

The change of the response latencies for the three treatment groups over the initial six sessions of training, and as a function of go and no-go trials, are depicted in Figure 3. In the first session, there was no apparent difference between go and no-go trials regardless of groups. However,

\section{STAGE 1: ACQUISITION}

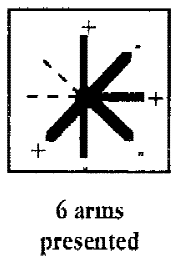

one by one

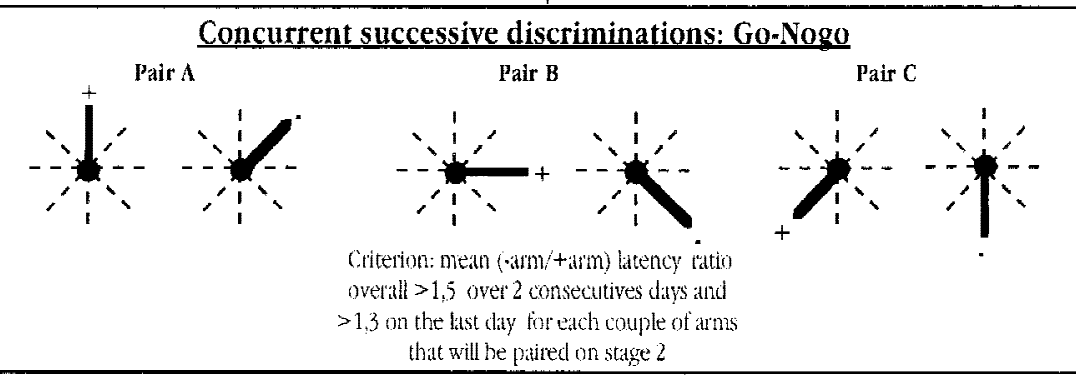

STAGE 2: MODIFIED PRESENTATIONS OF FAMILIAR ITEMS

Concurrent simultaneous discriminations: choices

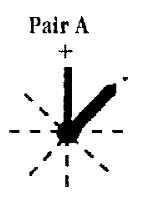

Pair B

Pair C

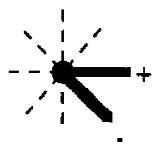

Figure 1 A diagrammatic illustration of the design of the experiment.

both S 17092 and tacrine affected the overall response latency to arm-entry. Whereas tacrine-treated mice took longer than vehicle controls to initiate an entry, S 17092treated mice were quicker. Furthermore, the response latencies were found to decrease progressively in the tacrine group and to a lesser extent in the vehicle group. In contrast, this measure remained relatively stable across the six first sessions in the S 17092 group. A 3-way ANOVA, with the between-subjects factor, Group and two within-subjects

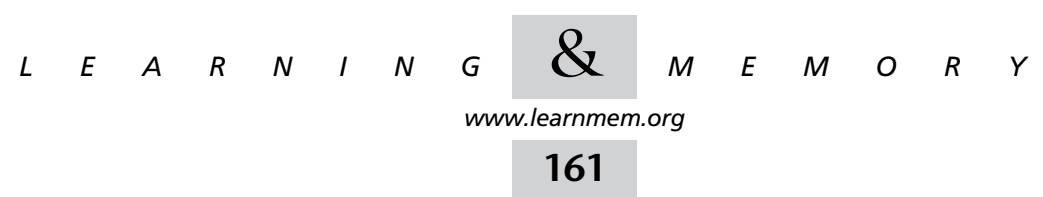




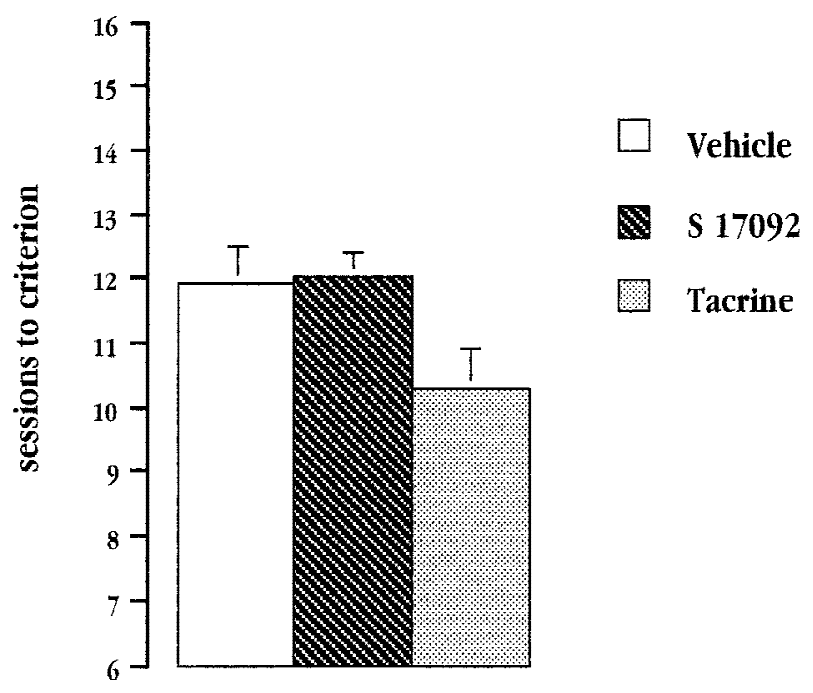

Figure 2 Mean number of sessions to criterion during successive go/no-go discrimination in Stage 1 for vehicle-treated aged controls, and tacrine- or S 17092-treated aged mice.

factors, Reinforcement (positive vs. negative arms) and Session, confirmed these impressions. It revealed a significant effect of Group $[\mathrm{F}(2,34)=23.70 ; P<0.001]$ and of Session $[\mathrm{F}(5,170)=9.60 ; P<0.001]$, as well as their interaction $[\mathrm{F}(10,170)=2.53 ; P=0.007]$. However, neither the main effect of Reinforcement nor its interactions attained significance (all F's $<1$ ). Furthermore, the main effect of Group remained significant when any one of the three groups was dropped from the ANOVA.

Over the final six sessions, there was a progressive increment in the difference on response latencies between go and no-go trials in all three groups, suggesting that all animals were able to discriminate between positive and negative arms as training progressed. These impressions were supported by the significant effect of Reinforcement $[\mathrm{F}(1,34)=59.59 ; P<0.001]$ and its interaction with Session $[\mathrm{F}(5,170)=8.37 ; P<0.001]$. Furthermore, the latency differential between go and no-go trials over the last four sessions appeared to be larger in tacrine-treated mice relative to vehicle controls. A similar but less pronounced trend was also seen in the S 17092 group. This impression was supported by the highly significant Group $\times$ Reinforcement interaction $[\mathrm{F}(2,34)=5.72 ; P=0.007]$

Further restrictive analysis suggested that this interaction stemmed mainly from the contrast between the tacrine and vehicle groups. The Group $\times$ Reinforcement interaction remained significant when the S 17092 group was dropped from the ANOVA, but not when the tacrine group was removed.

Over the final six sessions of training, tacrine treatment continued to retard the overall latency to initiate an armentry, while the opposite effect associated with S 17092 treatment had disappeared. This was supported by post-hoc pair-wise comparisons (tacrine vs. vehicle or tacrine vs. S 17092, $P<0.001$; S 17092 vs. vehicle, n.s.).

\section{Run Time}

Analyses were also performed on the run time measure (defined as the time necessary to run from the entrance to the food well of an arm) on go and no-go trials. Performance over the first six sessions and the last six sessions was analyzed separately.

Over the first six sessions (Fig. 4A), there was no difference in run times between go and no-go trials (Reinforcement and Reinforcement $\times$ Session: both F's $<1$, n.s.) but there was a significant decrease in the overall run time over the six sessions [Session: $\mathrm{F}(5,170)=33.33 ; P<0.001$ ] . This trend appeared to be similar across groups (Group $\times$ Session: $\mathrm{F}<1$ ). Both tacrine and S 17092 tended to slightly
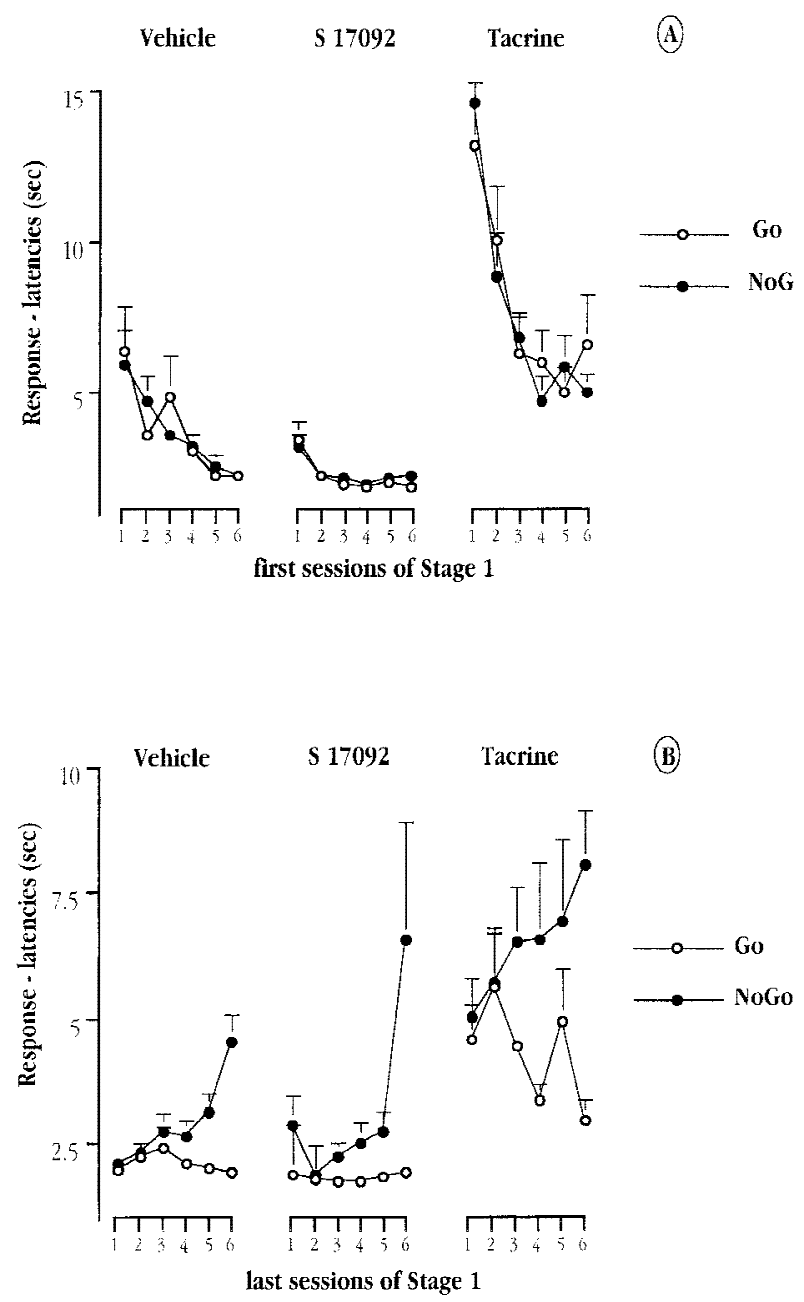

Figure 3 Mean response latency (in sec) to enter the positive (go trials) and negative arms (no-go trials) over the first six $(A)$ and the last six $(B)$ sessions of training prior to reaching criterion in Stage 1, for each of the three groups of aged mice: vehicle, tacrine, and $\mathrm{S}$ 17092.

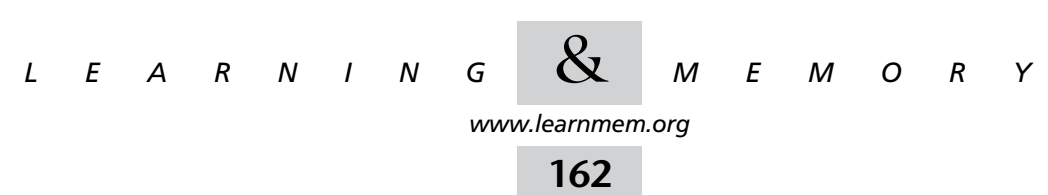



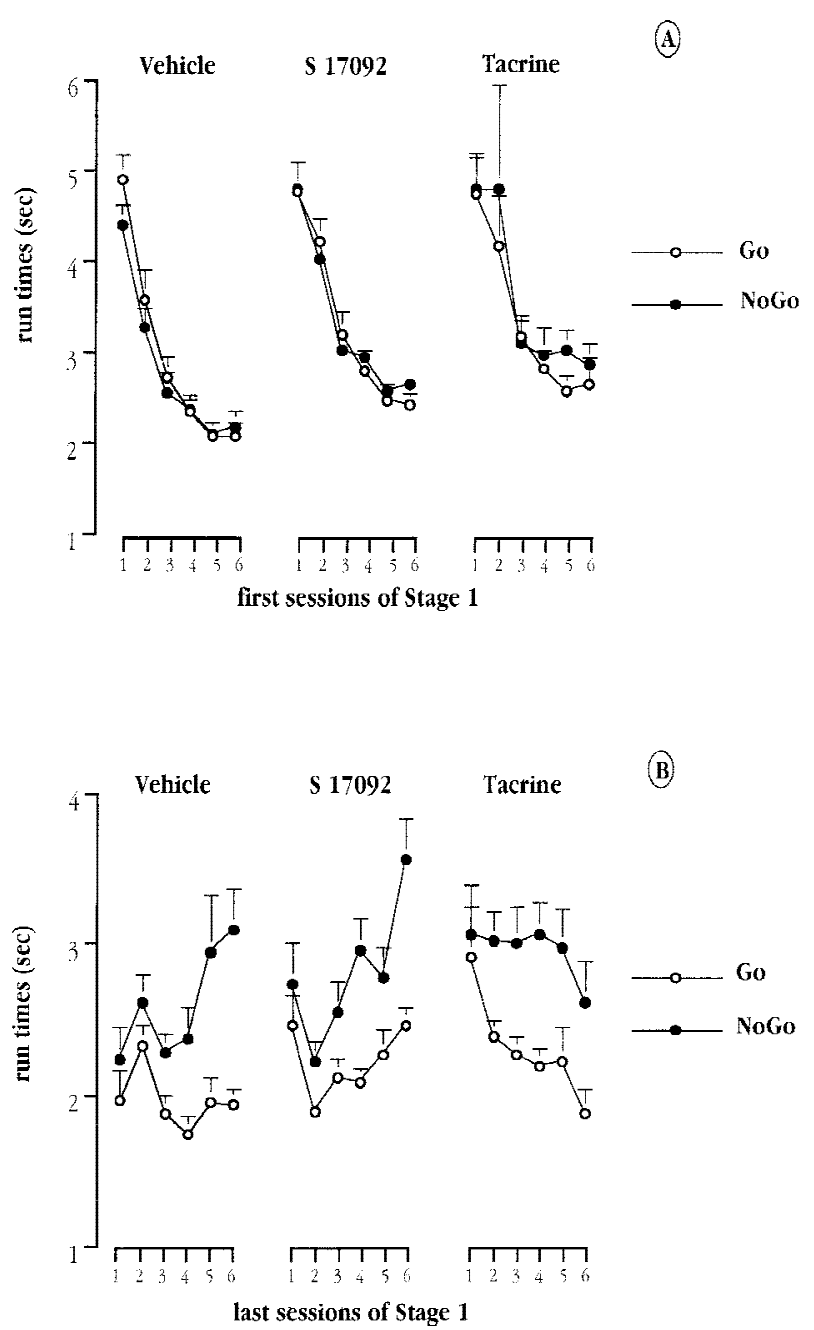

Figure 4 Mean run time (in sec) in positive (go trials) and negative (no-go trials) arms over the first six $(A)$ and the last six $(B)$ sessions of training in Stage 1, for each of the three groups of aged mice: vehicle, tacrine, and S 17092.

increase the overall run time but this failed to yield a significant Group effect $[\mathrm{F}(2,34)=2.79 ; P=0.075]$.

Over the last six sessions (Fig. $4 \mathrm{~B}$ ), the run time on no-go trials was slower than on go trials, and this difference was increased as training progressed. This gave rise to a main effect of Reinforcement $[\mathrm{F}(1,34)=61 ; P<0.001]$ and its interaction with Session $[\mathrm{F}(5,170)=9.16 ; P<0.001]$. The run time differential between go and no-go trials was of similar magnitude among groups (Group $\times$ Reinforcement: $F<1$ ). In these final sessions of Stage 1 , there was also no evidence for any between-group difference in terms of overall run speed (Group: $P>0.15$ ).

\section{Summary}

S 17092 treatment did not significantly affect the acquisition of successive go-no-go discriminations. Neither the rate of acquisition nor the level of discrimination performance was modified in terms of response latency or run time. The only clear effect of S 17092 was to reduce overall response latency in the first six sessions.

Conversely, tacrine showed some beneficial effects on performance. Tacrine-treated mice attained criterion performance earlier (i.e., with fewer sessions) and their discriminative performance as measured by the differential in armentry latencies between go and no-go trials in the final sessions was also enhanced. However, it should be noted that the latter effect might be partly attributed to the nearasymptotic level (of go latencies) seen in the other groups (but not in the tacrine group) at this phase of training. The discriminative performance reflected in the absolute differential in arm-entry latencies in the vehicle and S 17092 groups might have been underestimated due to this floor effect.

\section{Stage 2 \\ Choice Accuracy}

When required to make an explicit choice between two arms of opposing valence that were previously experienced separately, vehicle-treated aged mice failed to show any preference for the positive arm. They performed at a level close to chance with a mean ( \pm S.E.M.) percent correct of $53.00 \pm 2.84 \%$ in the first session and $58.33 \pm 2.99 \%$ in the second session. On the other hand, mice treated with $\mathrm{S}$ 17092 or tacrine were performing at a superior level (see Fig. 5). S 17092 group was performing at $68.80 \pm 4.19 \%$ and $72.50 \pm 2.11 \%$, whereas the tacrine group was performing at $65.90 \pm 3.27 \%$ and $66.2 \pm 4.03 \%$, in the first and second sessions, respectively.

Further pair-wise comparison indicated that the performance of S 17092-treated mice was significantly above that

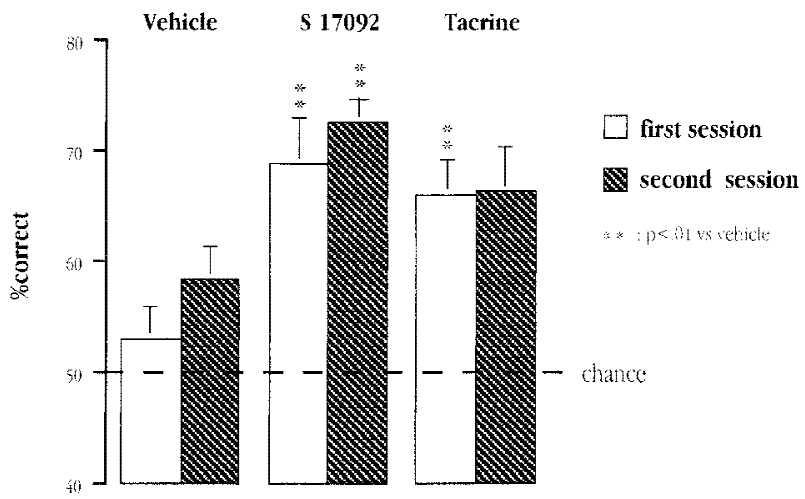

Stage 2

Figure 5 Mean percent correct on each of the two sessions of Stage 2 (three-pair simultaneous discrimination) for each of the three groups of aged mice: vehicle, tacrine, and S 17092.

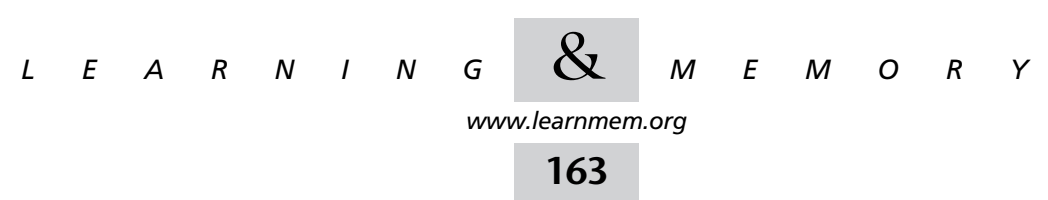




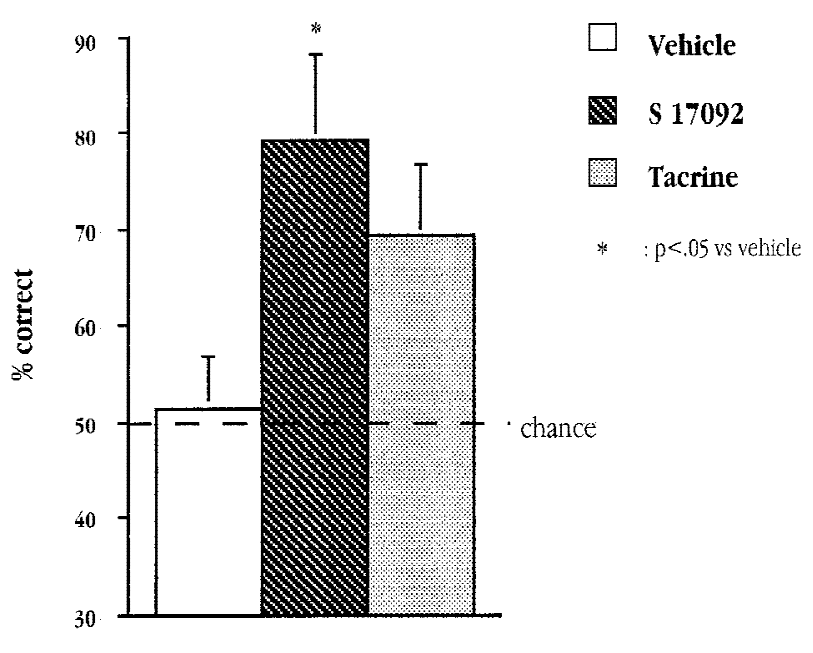

First trials of Stage 2

Figure 6 Mean percent correct on the three first trials of Stage 2 (initial exposure to each of the three pairs: A, B, and C) for each of the three groups of aged mice: vehicle, tacrine, and S 17092.

of vehicle-aged controls on both sessions $(P=0.004)$. Although, the present experiment did not consist of a group of young mice, it is interesting to note that our S 17092treated mice were performing at a level comparable to younger untreated animals (75.1\%) from an earlier experiment with identical design (see Fig. 5 of Marighetto et al. 1999). Conversely, the enhancing effect of tacrine was less striking, only attaining significance in the first session $(P=0.006$ vs. vehicle) but not in the second session $(P=0.13$ vs. vehicle).

A 2-way (Group $\times$ Session) ANOVA on choice accuracy only yielded a significant effect of Group $[\mathrm{F}(2,34)=6.62$; $P=0.004]$. This supported the conclusion that both drugs were effective in enhancing choice accuracy in Stage 2.

Supplementary analyses were performed separately on the choice accuracy on the first three trials of the first session. These trials are of particular interest because they comprised the first presentation of each of the three pairs (A, B, and $\mathrm{C}$ ), i.e., when these pair-wise arrangements were presented to the mice for the very first time.

Analysis of these trials would enable us to determine whether the enhancing effects of the drugs on choice accuracy described above was attributable to improved acquisition of pair discrimination as such, rather than via a flexible deployment of knowledge (individual arm's reward valence) acquired during Stage 1 . If so, performance on these initial trials should be close to chance level. However, if the enhancing effects 17092. of the drugs were already apparent in these initial trials, it would support the interpretation that they facilitate flexible transfer of past experience in novel situations.

As shown in Figure 6, the beneficial effects of both drugs on choice accuracy were already visible on these initial pair-wise discrimination trials (mean \pm s.E.M.: 69.21 $\pm 6.5 \%$ for tacrine, $79.25 \pm 8.6 \%$ for $\mathrm{S} 17092$, and 51.47 $\pm 6.26 \%$ for vehicle). This was confirmed by a one-way ANOVA that yielded a significant effect of Group $[\mathrm{F}(2,34)=3.91 ; P=0.03]$. However, pair-wise comparisons further revealed that this enhancing effect was only significant for S 17092 treatment $(P=0.044$ vs. vehicle) but not for tacrine $(P=0.159$ vs. vehicle).

\section{Response Latency}

Figure 7 depicted the response (arm-entry) latency expressed according to whether the response was directed to rewarded (correct response) or non-rewarded (incorrect response) arms as a function of treatment and session. The response latency for go trials and no-go trials in the last session of Stage 1 is also shown in Figure 7 to illustrate the effect associated with the transfer from Stage 1 to Stage 2 on this measure. It can be seen that tacrine treatment again led selectively to a global increase in the latency to initiate an arm entry.

An ANOVA with the factor Group and the two withinsubjects factors, Reinforcement (whether the chosen arm was positive or negative) and Session, was performed on the latency measures obtained in the two sessions of simultaneous 2-choice discrimination testing. This revealed a main effect of Group $[\mathrm{F}(2,34)=4.99 ; P=0.013]$ which stemmed mainly from a significant difference between tacrine and vehicle groups $(P=0.009)$. The other pair-wise comparisons failed to attain significance (tacrine vs. S 17092: $P=0.142$; vehicle vs. S 17092: $P=0.15$ ].

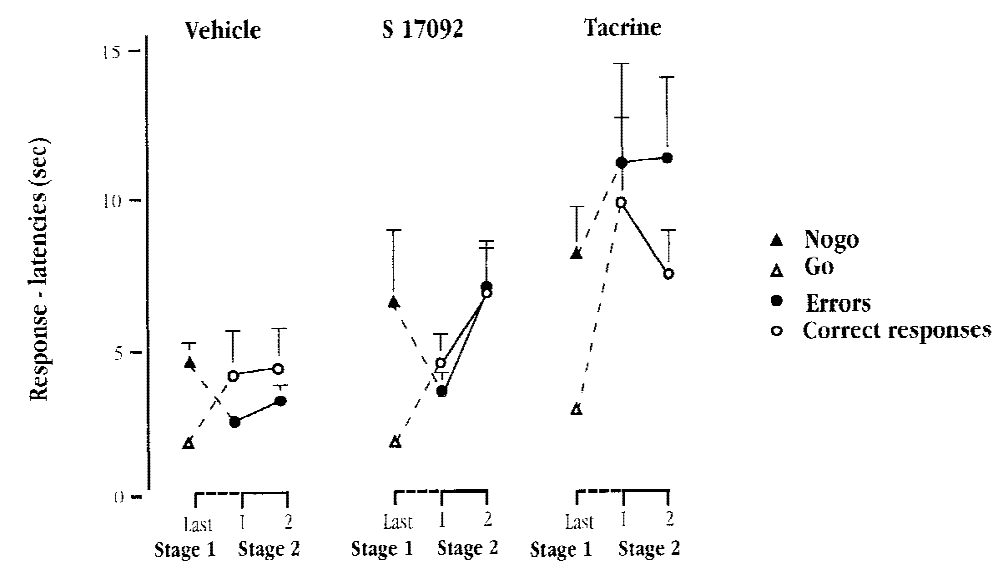

Figure 7 Mean latencies (in sec) to enter the positive and the negative arms (i.e., go vs. no-go trials in stage 1 , and correct responses vs. errors in stage 2) over the last session of Stage 1 and the two sessions of Stage 2 (three-pair simultaneous discriminations) for each of the three groups of aged mice: vehicle, tacrine, and $\mathrm{S}$

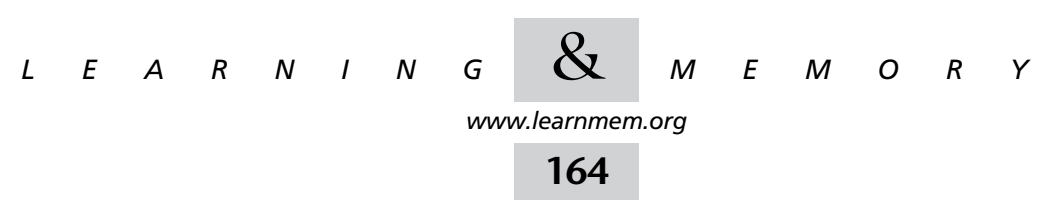


This is consistent with the previous (Stage 1) conclusion that tacrine significantly increased response latency. As shown in Figure 7, tacrine also appeared to modify the pattern of response latency as a function of whether the response was correct or not. This impression, however, only received tentative statistical support, with the Group $\times$ Reinforcement interaction approaching, but failing to attain significance $[\mathrm{F}(1,34)=3.05 ; P=0.06]$. No other effect was statistically significant.

\section{Run Time}

The run time measure obtained in Stage 2 was submitted to an ANOVA with the between-subjects factor Group and the two within-subjects factors, Reinforcement and Session (see Fig. 8). This revealed a main effect of Group $[F(2,34=3.5$; $P=0.04]$. Regardless of whether the chosen arms were rewarded or not, S 17092 increased the run time relative to vehicle $(P=0.006)$.

The main effect of Session $[\mathrm{F}(1,34)=7.5 ; P=0.01]$ and of Reinforcement $[\mathrm{F}(1,34)=49.8 ; P<0.001]$ as well as their interaction $[\mathrm{F}(2,34)=4.4 ; P=0.045]$ were also significant. The two main effects were associated with a reduction of run time as a function of Session, and overall shorter run time into positive arms relative to negative ones. The interaction effect arose because the effect of Reinforcement was increased from the first to second session. There was no evidence that these effects were differentially expressed between groups as there was no significant interaction terms involving Group.

\section{Summary}

In agreement with our previous data in normal (undrugged) mice (Marighetto et al. 1999), the present results demonstrated that vehicle-treated aged mice successfully discrimi-

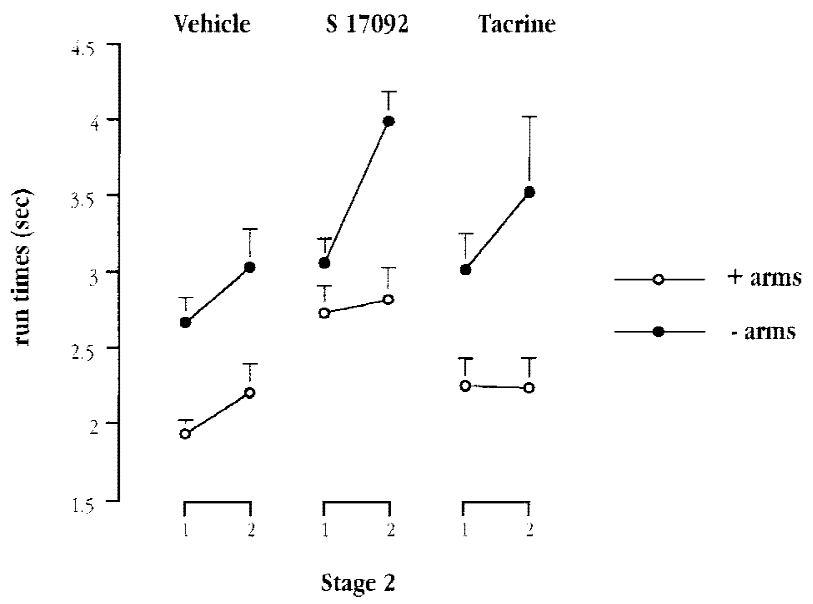

Figure 8 Mean run time (in sec) in positive (correct responses) and negative arms (incorrect responses) over the two sessions of Stage 2 (three-pair simultaneous discriminations) for each of the three groups of aged mice: vehicle, tacrine, and S 17092. nate between arms of opposing valence when these are presented one by one, in a successive go-no-go procedure. This was demonstrated in the final sessions of Stage 1, by the increased readiness of the mice to enter positive arms relative to negative ones (response latencies analysis). Their discriminative performance was also reflected in the runtime differential. However, they failed to translate their preference for the positive arms to correct choices in subsequent simultaneous discriminations (Stage 2).

The main result of this study is that chronic treatment of either S 17092 (10mg/kg per os) or tacrine (3mg/kg s.c.) significantly enhanced choice accuracy (percent correct analysis) relative to controls in Stage 2, although the effects of the two drugs on 2-choice performance were not identical, particularly on the very first trials. Furthermore, the procognitive effect on choice accuracy was not consistently accompanied by a similar effect on discriminative indices based on response latency or run-time. Specifically: (1) tacrine also increased the acquisition speed of go-no-go discrimination as reflected on the no-go/go ratio of arm-entry latencies in Stage 1, whereas S 17092 exhibited no such effect, (2) both tacrine and S 17092 failed to affect the run-time differential in both Stage 1 and Stage 2, despite the fact that this measure of discrimination was altered in control-aged mice, particularly at Stage 2 (Marighetto et al. 1999).

Finally, tacrine consistently increased overall response latency, an effect that persisted throughout the entire experiment, and was particularly pronounced in novel or changed situations (i.e., the initial sessions of Stage 1 or Stage 2). In contrast, an opposite effect of S 17092 on response latency was clearly seen only in the initial sessions of Stage 1. Furthermore, while tacrine failed to affect the overall run time along the arms, S 17092 increased it, even though the latter effect was only apparent in Stage 2.

\section{DISCUSSION}

The deficit observed in control aged mice (see Marighetto et al. 1999 for a direct comparison between aged and younger adult mice) was specific for one form of memory expression for acquired spatial discriminations. Successful discrimination between arms of opposing valence was obtained when these arms were presented one at a time in a successive go-no-go procedure. This was reflected in the subject's relative response (arm-entry) latency and run time between positive and negative arms. Yet, such demonstrable acquired knowledge failed to guide the aged mice towards the positive arm when confronted with an explicit choice between two arms. In the latter test condition, the acquired knowledge remained undetectable in the subject's choice behavior until the subject has committed an entry. Indeed, once the mouse has entered an arm, its run speed was affected by the valence of the arm as it was in Stage 1, although this form of memory expression was also found to

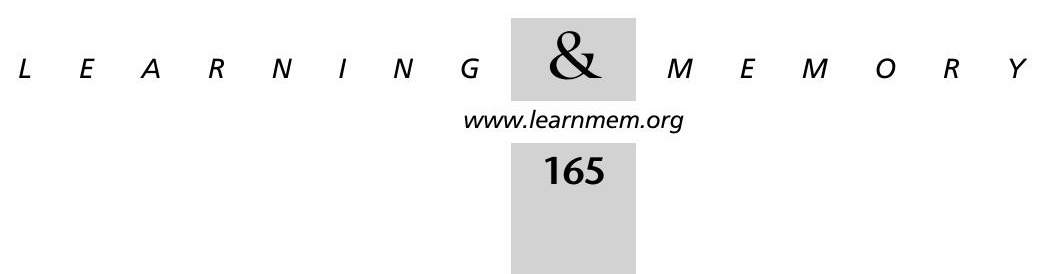


be reduced in aged mice as compared to younger adults when assessed in choice situations (Marighetto et al. 1999). Based on the theoretical interpretation offered by Eichenbaum et al. (1992, 1994), we have previously suggested that this kind of mnemonic inflexibility seen in the aged mice could be linked to an alteration in the relational processing of incoming/stored information. According to this, the aged mice stored or encoded the experiences during the first learning stage as separate, and therefore, impoverished representations of arm entries. Hence, they lacked the representation of a complex framework incorporating the relative relationships among arms, which normally enables comparisons and contrasts between separately experienced items. This is why they could perform normally when the test situations encourage the storage and use of separate representations of individually experienced items (successive go-no-go discriminations of arms), but failed in test situations that emphasize a judgement of the relative valence between two arms (which involves a comparison between two separate representations). The selectivity of the age-related deficit was therefore in coherence with the relational hypothesis that the system which sustains the acquisition of complex associations among items (relational representations) is dissociated from the one that enables the memorization of separate representations of information based on simple (stimulus-response or stimulus-reward) associations. By showing that choice performance of aged mice could be improved with or without a concomitant enhancement of the successive go-no-go discrimination, the present comparison of the effects associated with tacrine and S 17092 further support this hypothesis.

More specifically, it appeared that S 17092 enhanced choice accuracy in Stage 2 without inducing major changes on other performance indices in Stage 1 or in Stage 2. This led to the conclusion that this drug specifically improved flexible deployment of passed experiences. This interpretation is further supported by the fact that the beneficial effect of S 17092 was readily visible and statistically significant in the initial trials of Stage 2. Within the theoretical framework of relational memory, this flexible deployment of memories can be seen as a result of the acquisition of complex associations among separately experienced items.

In contrast, tacrine affected discriminative performance at both stages of the experiment and its beneficial effect on choice accuracy was not significant in the first trials of Stage 2 . These cannot be interpreted as a specific improvement of the flexible deployment of memories in novel situations. This less-specific effect of tacrine might be taken as evidence for the hypothesis that acetylcholine plays a general role in sensory information processing and attention (for review, see Blokland 1996; Sarter and Bruno 1997). This latter interpretation is further supported by the observation that overall response latencies were increased in tacrine-treated mice, and in particular, in novel or changed situations. Given that tacrine did not affect run time, it appeared that the drug specifically retarded response initiation but not response execution. This suggested that the drug had no effect on locomotion but affected attention or behavioral inhibition processes. Interestingly, S 17092 was effective in modifying the overall response latency in the initial sessions of Stage 1, but the direction of this effect was opposite to that of tacrine. The possible mechanisms that might underlie such a disinhibiting effect are unknown. This is further complicated by the fact that S 17092 slowed down the run speed in Stage 2 . Nevertheless, the observation that the two drugs exerted opposite effects on the time parameters while at the same time are both effective in enhancing choice accuracy demands further considerations. Firstly, the effects of tacrine can be seen as evidence for a positive effect on attention that can lead to enhanced choice performance on Stage 2 by aged mice. Secondly, the equally beneficial effect of S 17092 on choice accuracy in Stage 2 indicates that the hypothesized effect on attention of tacrine is not a necessary condition for the amelioration of the specific cognitive impairment experience by our aged mice. Hence, it might be suggested that the two drugs are targeting different neurobiological processes, acting as cognitive enhancers via distinct cognitive processes.

We have observed previously that aging did not alter the go-no-go discriminative performance as measured by response-latency differential, but tended to reduce the differential in run times between positive and negative arms (Marighetto et al. 1999). This latter index was also affected by aging in the 2-choice condition in Stage 2 . Our results showed that tacrine enhanced the go-no-go differential in terms of response latency but not in terms of run time, whereas both indices remained unaffected by S 17092 . Hence, it is tempting to speculate that the cognitive system that underlies the discriminative performance as measured by run time is, at least partially, dissociable from the one underlying the performance revealed by arm-entry latency. On the one hand, the progressively increasing divergence between the latency to enter positive and negative arms could be considered as following a progressive increment in stimulus-response associations in the form of: "when this particular arm is opened $\rightarrow$ Go" and "when this one is presented $\rightarrow$ No go ," the progressive increase in the differential run time between positive and negative arms can be viewed as a result of the progressive formation of stimulusreward associations in the forms of "whenever I am in this particular arm, I would get a food reward," and "whenever I am in that arm, I would not be rewarded."

When our study comparing tacrine and S 17092 and the previous report (Marighetto et al. 1999) comparing normal aged and adult mice are considered together, one might speculate the existence of three distinct behavioral expressions of acquired knowledge concerning the reward va-

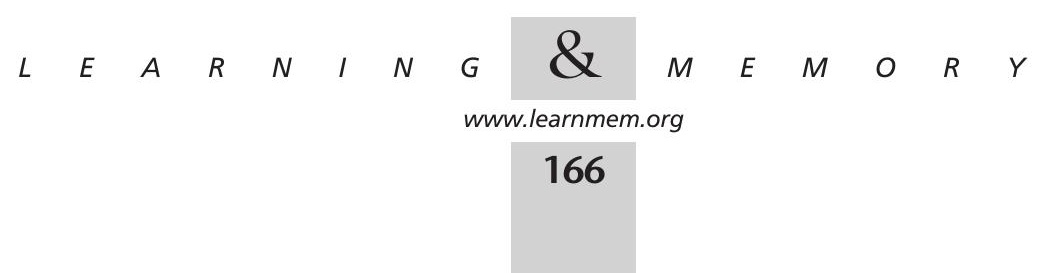


lence of individual arms as demonstrated through such a spatial discrimination paradigm. These are represented by (1) the percentage of correct choices in simultaneous discriminations; (2) the relative readiness to make an entry in the successive discrimination procedure; and (3) the relative run speed toward the food well in both procedures.

The different expressions of 2-arm discriminations revealed by the effects of aging and those of drug administration are reminiscent of a triple dissociation of memory systems revealed through a comparative analysis among the effects of hippocampal, striatal, and amygdalar lesions (McDonald and White 1993). Hence, one can speculate that these aspects of mnemonic performance might rely on separate and more or less independent memory systems. The "relational" memory system, sustaining the ability to compare information originating from separate sources, and hence 2-choice discrimination performance, would be impaired by aging but enhanced by tacrine or S 17092. The "habit" memory system, acquiring stimulus-response associations revealed in relative response latencies, would be unaffected by aging and S 17092 but sensitive to the effects of tacrine. The "affective" memory system, sustaining the formation of stimulus-reward associations reflected in run speed, would be largely preserved in aged mice and unaffected by either procognitive agents studied here, although its expression can be compromised in certain conditions (Marighetto et al. 1999). Further studies are needed to test the validity of these hypotheses. A lesion approach would be particularly suited to address these issues concerning the critical anatomical substrates underlying these hypothetically distinct memory systems.

\section{MATERIALS AND METHODS}

\section{Subjects and Drugs}

Subjects were 48 naive mice of the $\mathrm{C} 57 / \mathrm{bl6}$ Jico inbred strain obtained from IFFA Credo (Lyon, France). They were between the ages of 23 and 25 months at the beginning of the experiment. On arrival, the mice were caged in groups and housed in a climatized animal-keeping room, maintained under a 12:12 light-dark cycle and ad lib food and water. After 4-6 weeks, they were caged singly. Pharmacological treatment commenced a fortnight later.

The mice were divided into three treatment groups: vehicle $(n=18)$, tacrine $(n=18)$ and S $17092(n=12)$. Preliminary experiments indicated that tacrine treatment was associated with higher between-subjects variability. Thus, more subjects were included in the tacrine group than in the $S 17092$ one. In the course of the experiment, eight mice died ( 1 vehicle, 4 tacrine, and 3 S 17092) and three others (2 vehicle and 1 S 17092) were dropped from further behavioral training (see Behavioral Procedure below). The final group sizes were: vehicle $(n=15)$, Tacrine $(n=14)$ and $S$ $17092(n=8)$.

S 17092 , at a dose of $10 \mathrm{mg} / \mathrm{kg}$, was administered orally whereas tacrine, at a dose of $3 \mathrm{mg} / \mathrm{kg}$, was administered subcutaneously. Mice in the vehicle condition, received $10 \mathrm{ml} / \mathrm{kg}$ of saline either orally $(n=9)$ or subcutaneously $(n=9)$. These relevant drug doses and the chosen routes of administration were established on the basis of previous reports on the procognitive effects of these compounds (for S 17092: Lépagnol et al. 1996; Lestage et al. 1998b; for tacrine: Flood and Cherkin 1988; Itoh et al. 1990; Riekkinen et al. 1991; Kirkby et al. 1996; Pavone et al. 1998). Tacrine ( 9 amino1,2,3,4-tetrahydroacridine hydrochloride: hydrate) was obtained from Sigma Aldrich (St. Quentin Fallavier, France, product no. A3773). S 17092 was obtained from the Institut de Recherches Internationales SERVIER (IRIS) (Courbevoie, France).

Treatment began one week prior to the beginning of behavioral training. In the first week, the mice were treated daily at $\sim 1600 \mathrm{hrs}$. From the first day of behavioral training, treatment was administered either $45 \mathrm{~min}$ (for tacrine and placebo-oral groups) or $1 \mathrm{hr}$ (for S 17092 and vehicle-subcutaneous) prior to behavioral training.

Food deprivation was introduced progressively over the three days prior to the beginning of behavioral testing. The animals were eventually given a fixed amount of laboratory food chow per day, and their body weight was maintained at a stable level, $~ 90 \%$ of the free feeding weight.

Experiments were carried out in accordance with the European Communities Council Directive of November 24, 1986 (86/ 609/EEC).

\section{Apparatus}

The apparatus was a fully automated, elevated, 8-arm radial maze, located in a quiet testing room enriched with distal cues. Its dimension and construction have been described in full elsewhere (Marighetto et al. 1999). A door was mounted at the entrance to each arm, and a pellet dispenser was installed at the end of each arm. Door movements were controlled by a computer program which also tracked the position of the mouse within the maze continuously via pressure captors underneath the central platform and two pairs of photocell beams installed along each arm. One pair of photocell beams guarded the entrance of the arm, and one just in front of the food well. This enabled a real-time control of the accessibility to the maze arm(s) according to a predetermined test schedule.

\section{Behavioral Procedure}

\section{Shaping}

The animals were first habituated to the maze over a period of two days. On each of these days, the animals were allowed to move freely in the maze individually until they had collected a reward from each of the eight prebaited arms. Subjects that failed to collect all the food within $15 \mathrm{~min}$ on the second day were given one additional session on the same day. Subjects that failed to collect all the food during this extra session within 15 min were dropped from further testing.

\section{Discrimination Tasks}

Each subject was separately assigned six adjacent arms. Out of these, three served as positive (baited) arms, and the remaining three as negative (not baited) arms. The relative locations of these arms were such that these six arms could be grouped into three pairs of adjacent arms with opposing valence (see in Figure 1, pairs $A, B, C$ ). In addition, it was ensured that if the positive arms of Pairs $\mathrm{A}$ and $\mathrm{B}$ were on the left, the positive arm of pair $\mathrm{C}$ had to be on the right, and vice versa.

As shown in Figure 1, the present experiment consisted of a two-stage paradigm. The only parameter that was changed from Stage 1 to Stage 2 was the way in which the arms were presented

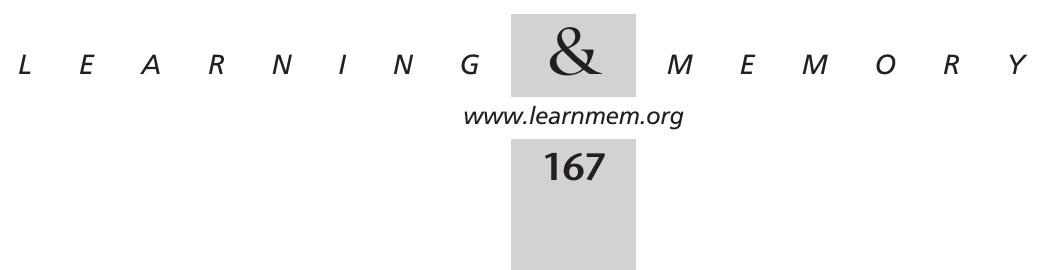


to the mice. As detailed below, the arms were presented one at a time in Stage 1 and in pairs in Stage 2.

\section{Stage 1}

In Stage 1, the six arms were always presented one at a time using a go-no-go discrimination procedure. On each trial, the door to one arm (which could be a positive or a negative one) was open, and remained so for a maximum duration of $120 \mathrm{sec}$; this was later reduced to $20 \mathrm{sec}$ as training progressed. If the subject entered and reached the food well within the allotted time, the door would close as soon as they returned to the central platform. If the subject failed to enter in time, the door would close. If the subject entered the arm but failed to reach the food well in time, the door would close as soon as it returned to the central platform. In all cases, the trial ended as the door closed, and the mouse was confined to the central platform for $5 \mathrm{sec}$, which constituted the inter-trial interval.

Each daily session consisted of 24 trials with four presentations of each of the six arms according to a pseudo-random sequence.

Training continued until the mice attained criterion performance (as specified below) with respect to each of the three predetermined pairs of arms (Fig. 1, pairs A,B,C). All mice must undergo a minimum of six daily sessions in Stage 1, and mice failing to reach criterion performance in 16 sessions were dropped from further testing. When a mouse attained criterion performance at this stage, it was transferred to Stage 2 testing on the following day.

Two distinct performance indices were used to evaluate the discriminative performance in Stage 1. The first index was based on response latency, defined as the time elapsed from the beginning of the trial (opening of one door) to the moment when the mouse interrupted the photocell-beam at the entrance of the arm. This first index was also mentioned as arm-entry latency. The second index was based on run time, defined as the time elapsed between the interruption of the photocell beam at the arm entrance and interruption of the second photocell beam located in front of the food well. For each predetermined pair of arms of opposing valence (pairs A,C) discriminative performance was measured by: (1) the difference between the medial response latency (per session) to enter the negative arm relative to the positive arm; and (2) the equivalent difference based on run time. Whereas the latter measure enabled us to evaluate the animals' behavior after the initiation of an approach response, the former measure corresponded more closely to the decision-making process of the approach response.

The criterion for transfer from Stage 1 to Stage 2 was based on a ratio between response latency for the negative arm and response latency for the positive arm. This "discriminative ratio" contrasts the readiness of a subject to enter negative arms relative to positive ones. A ratio of unity represents chance performance or no preference. When this ratio is well above unity, it indicates that the subject is more ready to enter the positive arms and/or more reluctant to enter the negative ones. A mouse was considered to have attained criterion performance when its overall discriminative ratio was $>1.5$ for two consecutive sessions, and provided that the discriminative ratio of each of the three adjacent pairs $(A, B, C)$ was $>1.3$ on the last session of Stage 1 .

\section{Stage 2}

This lasted for two consecutive days. The discrimination problems presented in Stage 2 were between the same six arms as those in Stage 1 and the reward valence of each arm also remained unchanged. However, their presentation was modified, namely, the six arms were now grouped into three pairs.
In each trial, the subject was confronted with access to two adjacent arms with opposing valence as depicted in Figure 1 as pairs $\mathrm{A}, \mathrm{B}$, or $\mathrm{C}$. A choice was considered to be made when the subject had reached the food well of an arm; this also triggered the closure of the door to the alternative arm. The trial was finished as soon as the subject returned to the central platform. As in Stage 1, the inter-trial interval was $5 \mathrm{sec}$. Each daily session consisted of 20 consecutive trials comprising of alternating presentations of pairs $\mathrm{A}, \mathrm{B}$, and $\mathrm{C}$ according to a pseudo-random sequence.

Again, two distinct behavioral indices were used to evaluate discriminative performance. The first measure was choice accuracy in terms of percent correct. The second was the difference between the median run time in the positive and negative arms. Whereas the first measure more closely referred to the decisionmaking process of an approach/avoidance response, the second measure enabled one to evaluate the animals' behavior after the initiation of an approach response. In addition, the second index also allowed a direct comparison with Stage 1 (successive go-no-go discrimination) performance because the same index was also obtained in Stage 1.

Although they were not used as an index of discriminative performance at this stage, response latencies were also recorded, and still analyzed as a function of whether the chosen arm was positive or negative.

\section{Statistical Analysis}

Data were analyzed by analyses of variance (ANOVAs). Each ANOVA always included a between-subject factor, Group, which contrasted the three treatment conditions. Post-hoc pair-wise comparisons were performed using the Scheffe's procedure.

\section{ACKNOWLEDGMENTS}

This work was supported by the Centre National de la Recherche Scientifique and the Institut de Recherches Internationales Servier. We are very grateful to Dr. Jacques Micheau and Dr. Benjamin K. Yee for their helpful comments on previous versions of the manuscript.

The publication costs of this article were defrayed in part by payment of page charges. This article must therefore be hereby marked "advertisement" in accordance with 18 USC section 1734 solely to indicate this fact.

\section{REFERENCES}

Bartus, R.T., R.L. Dean, M.J. Pontecorco, and C. Flicker. 1985. The cholinergic hypothesis: A historical overview, current perspective, and future directions. Ann. NY Acad. Sci. 444: 332-358.

Beal, M.F. and M.F. Mazurek. 1987. Substance P-like immunoreactivity is reduced in Alzheimer's disease cerebral cortex. Neurology 37: 1205-1209.

Bennett, G.W., T.M. Ballard, C.D. Watson, and K.C.F. Fone. 1997. Effects of neuropeptides on cognitive function. Exp. Gerontol. 32: 451-469.

Blokland, A. 1996. Acetylcholine: A neurotransmitter for learning and memory? Brain Res. Rev. 21: 285-300.

Buck, S.H., P.P. Deshmukh, T.F. Burks, and H.I. Yamamura. 1981. A survey of substance $\mathrm{P}$, somastotatin, and neurotensin levels in aging in the rat and human central nervous system. Neurobiol. Aging 2: 257-264.

Cohen, N.J. 1984. Preserved learning capacity in amnesia: Evidence for multiple memory systems. In Neurospsychology of memory (ed. L.R. Squire and N. Butters), pp. 83-103. Guilford Press, New York, NY.

Coyle, J.T., D.L. Price, and M.R. DeLong. 1983. Alzheimer disease: A disorder of cortical cholinergic innervation. Science 219: 1184-1190.

Davies, P. and A.J.F. Maloney. 1976. Selective loss of central cholinergic neurons in Alzheimer's disease. Lancet 2: 1403-1046.

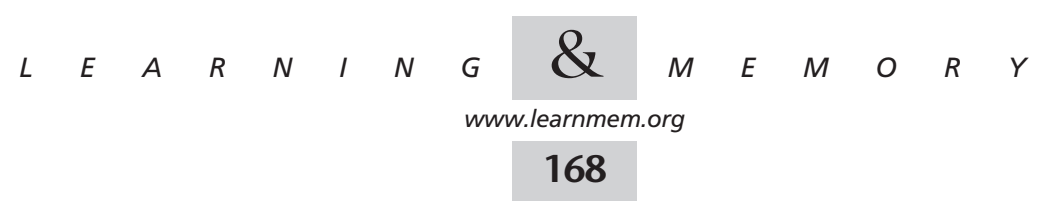


Dekker, A., W.H. Gispen, and D. De Wied. 1987. Axonal regeneration, growth factors and neuropeptides. Life Sci. 41: 1667-1678.

Dupont, A., P. Savard, Y. Merand, F. Labrie, and J.R. Boissier. 1981. Age-related changes in central nervous system enkephalins and substance P. Life Sci. 29: 2317-2322.

Eichenbaum, H. 1992. The hippocampal system and declarative memory in animals. J. Cognitive Neurosci. 4: 217-231.

Eichenbaum, H., T. Otto, and N.J. Cohen. 1994. The hippocampus-What does it do? Behav. Neural Biol. 57: 2-36.

Flood, J.F. and A. Cherkin. 1988. Effect of acute arecoline, tacrine and arecoline+tacrine post-training administration on retention in old mice. Neurobiol. Aging 9: 5-8.

Ferrier, I.N., A.J. Cross, J.A. Johnson, G.W. Roberts, T.J. Crow, J.A. Corsellis, Y.C. Lee, D. O'Shaughnessy, T.E. Adrian, G.P. McGregor, et al. 1983. Neuropeptides in Alzheimer type dementia. J. Neurol. Sci. 62: $159-170$

Gabrieli, J.D.E. 1996. Memory systems analyses of mnemonic disorders in aging and age-related diseases. PNAS 93: 13534-13540.

Gallagher, M. and P.J. Colombo. 1995. Aging: the cholinergic hypothesis of cognitive decline. Curr. Opinion Neurobiol. 5: 161-168.

Giovannini, M.G., F. Casamenti, A. Nistri, F. Paoli, and G. Pepeu. 1991. Effect of thyrotropin releasing hormone (TRH) on acetylcholine release from different brain areas investigated by microdialysis. $\mathrm{Br}$. $\mathrm{J}$. Pharmacol. 102: 363-368.

Hasenöhrl, R.U., J.P. Huston, and T. Schuurman. 1990. Neuropeptide Subtance $\mathrm{P}$ improves water maze performance in aged rats. Psychopharmacol. 101: 23-26.

Husain, N.M. and C.B. Nemeroff. 1990. Neuropeptides and Alzheimer's disease. J. Am. Geriatr. Soc. 38: 918-925.

Itoh, J., T. Nabeshima, and T. Kameyama. 1990. Utility of an elevated plus-maze for the evaluation of memory in mice: Effects of nootropics, scopolamine and electroconvulsive shock. Psychopharmacol. 101: $27-33$.

Iwasaki, Y., M. Kinoshita, K. Ikeda, K. Takamiya, T. Shiojima. 1989. Trophic effect of various neuropeptides on the cultured ventral spinal cord of rat embryo. Neurosci. Lett. 101: 316-320.

Kirkby, D.L., D.N.C. Jones, J.C. Barnes, and G.A. Higgins. 1996. Effects of anticholinesterase drugs tacrine and E2020, the 5HT3 antagonist ondansetron, and the $\mathrm{H} 3$ antagonist thioperamide, in models of cognition and cholinergic function. Behav. Pharmacol. 7: 513-525

Lépagnol, J., C. Lebrun, P. Morain, G. de Nanteuil, and V. Heidet. 1996. Cognition enhancing effects of S 17092, a potent and long acting inhibitor of post-proline cleaving enzyme (PPCE). Soc. Neurosci. Abst. 142.

Lestage, P., A. Iris-Hugot, M.H. Gandon, and J. Lépagnol. 1998a Involvement of nicotinergic mechanisms in thyrotropin-releasing hormone-induced neurologic recovery after concussive head injury in the mouse. Eur. J. Pharmacol. 357: 163-171.

Lestage, P., C. Lebrun, F. Iop, A. Hugot, N. Rogez, P. Greve, D. Favale, M.H. Gandon, O. Rainbault, and J. Lépagnol. 1998b. S 17092-1, new post-proline cliving enzyme inhibitor: Memory enhancing effect and substance P neuromodulatory activity. In Behavioural Biology Advance . Progress in Alzheimer's and Parkinson's diseases. (ed. A. Fischer, I. Hanin, and M. Yoshida), pp. 653-660. Plenum Press, New York, NY.

Marighetto, A., N. Etchamendy, K. Touzani, C. Cortes-Torrea, B.K. Yee, J.N.P. Rawlins, and R. Jaffard. 1999. Knowing which and knowing what: A potential mouse model for age-related human declarative memory decline. Eur. J. Neurosci. 11: 3312-3322.

Mattioli, R., R.K.W. Schwarting, and J.P. Huston. 1992. Recovery from unilateral 6-hydroxydopamine lesion of substantia nigra promoted by the neurotachykinin substance $\mathrm{P}^{1-11}$. Neuroscience 48: 595-605.

McDonald, R.J. and N.M. White. 1993. A triple dissociation of memory systems: hippocampus, amygdala and dorsal striatum. Behav. Neurosci. 107: 3-22.

Mellow, A.H., T.S. Sunderland, R.M. Cohen, B.A. Lawlor, L.J. Hill, P.A. Newhouse, M.R. Cohen, and D.L. Murphy. 1989. Acute effects of high-dose thyrotrophin-releasing hormone infusions in Alzheimer's disease. Psychopharmacol. 98: 403-407.

Mellow, A.H., S.M. Aronson, B. Giordani, and S. Berent. 1993. A peptide enhancement strategy in Alzheimer's disease; Pilot study with TRH/physostigmine infusions. Biol. Psychol. 34: 271-273.

Mohammed, A.H. 1993. Effects of cholinesterase inhibitors on learning and memory in rats: A brief review with special reference to THA. Acta. Neurol. Scand. 49: 29-35.

Molchan, S.E., A.H. Mellow, J.L. Hill, H.J. Weingartner, R.A. Martinez, B Vitiello, and T. Sunderland. 1992. The effects of thyrotrophin-releasing hormone and scopolamine in Alzheimer's disease and normal volunteers. J. Psychopharmacol. 6: 489-500.

Molchan, S.E., A.H. Mellow, B.A. Lawlor, H.J. Weingartner, R.M. Cohen, M.R. Cohen, and T. Sunderland. 1990. TRH attenuates scopolamine-induced memory impairment in humans. Psychopharmacol. 100: 84-89.

Pavone, F., F. Capone, M. Battaglia, and M. Sansone. 1998. Shuttle-box avoidance learning in mice: Improvement by combined glucose and tacrine. Neurobiol. Learn. Mem. 69: 204-210.

Pitsikas, N., B.M. Spruijt, M. Josephy, S. Algeri, and W.H. Gispen. 1991. Effect of ORG 2766, an ACTH(4-9) analogue, on recovery after bilateral transection of the fimbria fornix in the rat. Pharmacol. Biochem. Behav. 38: 931-934.

Poon, L.W. 1985. Differences in human memory with aging: Nature, causes, and clinical implications. In Handbook of the psychology of aging (ed. J.Birren and K.W. Schaie ), pp. 427-462. New York, NY.

Portevin, B., A. Benoist, G. Rémond, Y. Hervé, M. Vincent, J. Lépagnol, and G. Denanteuil. 1996. New prolyl endopeptidase inhibitors: in vitro and in vivo activities of azabicyclo[2.2.2] octane, azabicyclo[2.2.1] heptane, and perhytroindole derivatives. J. Med. Chem. 39: 2379-2391.

Qizilbash, N., A. Whitehead, J. Higgins, G. Wilcock, L. Schneider, and M. Farlow. 1998. Cholinesterase inhibition for Azheimer disease: A meta-analysis of the tacrine trials. Dementia trialists collaboration. JAMA 280: 1777-1782.

Riekkinen, P.J.R., J. Sirvio, M. Riekkinen, and P. Riekkinen. 1991. Effects of THA on passive avoidance retention performance of intact, nucleus basalis, frontal cortex and nucleus basalis + frontal cortex-lesioned rats. Pharmacol. Biochem. Behav. 39: 841-846.

Rossor, M.N., P.C. Emson, D. Dawbarn, C.Q. Mountjoy, and M. Roth. 1986. Neuropeptide and dementia. Progr. Brain Res. 66: 143-159.

Sarter, M. and J.P. Bruno. 1997. Cognitive functions of cortical acetylcholine: Towards a unifying hypothesis. Brain Res. Rev 23: $28-46$.

Schugens, M.M., I. Daum, M. Spindler, and N. Birbaumer. 1997. Differential effects of aging on explicit and implicit memory. Aging, Neuropsychology and Cognition 4: 33-44.

Wang, Z.P., S.Y. Man, and F. Tang. 1981. Age-related changes in the contents of neuropeptides in the brain and pituitary. Neurobiol. Aging 15: 529-534.

Whitty, C.J., G. Kapatos, and M.J. Bannon. 1993. Neurotrophic effects of substance $\mathrm{P}$ on hippocampal neurons in vitro. Neurosci. Lett. 164: 141-144.

Wilk, S. 1983. Minireview on prolyl endopeptidase. Life Sci. 33: 2149-2157.

Received December 14, 1999; accepted in revised form April 5, 2000.

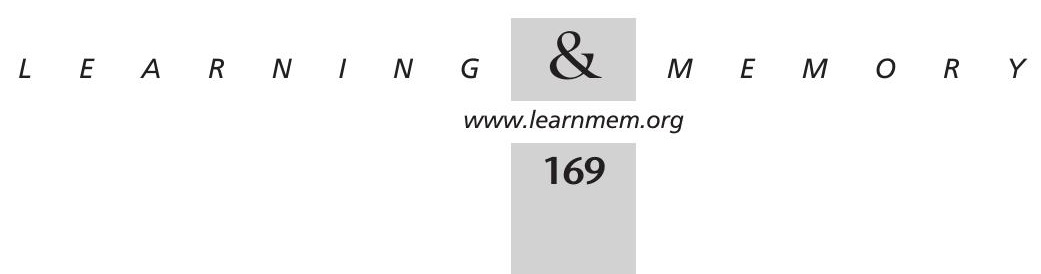



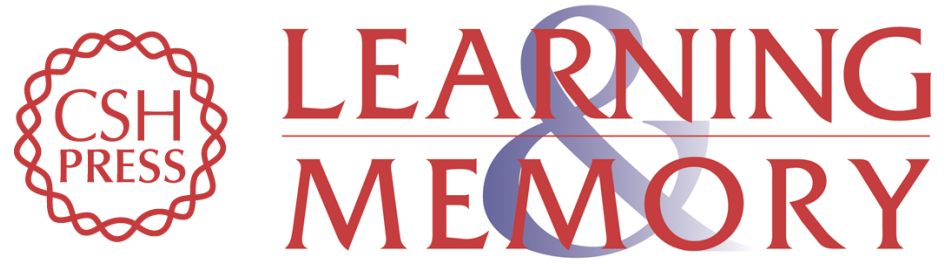

Further Evidence for a Dissociation Between Different Forms of Mnemonic Expressions in a Mouse Model of Age-related Cognitive Decline: Effects of Tacrine and S 17092, a Novel Prolyl Endopeptidase Inhibitor

Aline Marighetto, Khalid Touzani, Nicole Etchamendy, et al.

Learn. Mem. 2000, 7:

Access the most recent version at doi:10.1101//m.7.3.159

References

License

Email Alerting Service
This article cites 37 articles, 3 of which can be accessed free at: http://learnmem.cshlp.org/content/7/3/159.full.html\#ref-list-1

Receive free email alerts when new articles cite this article - sign up in the box at the top right corner of the article or click here. 